\title{
PAPR REDUCTION USING EFFICIENT PEAK CLIPPING TECHNIQUE IN OFDM SYSTEMS
}

\author{
Sarvjeet Kumar ${ }^{1}$, Shelly Chugh ${ }^{2}$, Jyoti Kohli ${ }^{3}$ \\ ${ }^{1}$ Assistant Professor, Dept. of ECE, S (P.G).I.T.M, Rewari, Haryana, India \\ ${ }^{2}$ Assistant Professor, Dept. of ECE, S (P.G).I.T.M, Rewari, Haryana, India \\ ${ }^{3}$ Assistant Professor, Dept. of ECE, LPU, Phagwara, Punjab, India
}

\begin{abstract}
Orthogonal frequency division multiplexing (OFDM) is the most popular modulation technique which is used for high speed communication system. It provides high spectral efficiency and low implementation complexity. However the main issue of OFDM is its high Peak to Average Power Ratio (PAPR) which results from sum of several sinusoidal signals. So the Efficient Peak Clipping Technique has used to reduce the peak-to-average power ratio (PAPR) of OFDM systems. By introducing different number of Subcarriers N, Up sampling factor k, Number of all samples n, Mapping Order M, and Phase Offset P, a desired level of PAPR has been achieved. At the same time by introducing the proposed technique better tradeoff between BER and SNR has been maintained.
\end{abstract}

Keywords-Peak-to-average power ratio (PAPR), Signal to noise ratio, (SNR), Complementary cumulative distribution function (CCDF), Bit error rate (BER), Orthogonal frequency division multiplexing (OFDM), Crest factor (CF)

\section{INTRODUCTON}

The demand of high data rate services has been increasing very rapidly and there is no probability of its slowdown. It means the requirement of high data rate is very high. For this purpose multicarrier modulation technique is used. In multicarrier modulation the most commonly used technique is orthogonal frequency division multiplexing (OFDM). OFDM is an efficient and marvelous technique which is widely used in modern wireless communication due to its high spectrum efficiency. So OFDM is a method of encoding digital data based upon multiple carrier frequencies In other words, OFDM is multi path multiplexing technique where large number of Orthogonal, Overlapping, and narrow band sub carriers transmitted in parallel. [4] In OFDM system high data rate transmission is divided into lower data rate and that are transmitted simultaneously over number of subcarriers. Each of these signal are individually modulated and transmitted over the channel and at the receiver, signal will be demodulated and recombine to recover the original signal. In OFDM System all sub carriers are arranged orthogonally. As per the advantage of OFDM system, it provides the better spectral efficiency. But, there is one critical drawback of OFDM based transmission systems, is the high peak-to-average power ratio (PAPR). It is defined as the maximum power occurring in the OFDM transmission to the average power of the OFDM transmission.

\section{BACKGROUND}

In OFDM System many problems are associated with it, they are phase noise, Image rejection and PAPR. But PAPR affect the system severally, due to which the efficiency of High Power Amplifier is reduced.

\subsection{PAPR}

It is known that an OFDM signal consists of or includes a large number of independently modulated subcarriers, which can give rise to a large Peak to Average Power Ratio (PAPR), when it is added up coherently. Whenever $\mathrm{N}$ equi-amplitude signals are added with the same phase, they produce a peak power that is $\mathrm{N}$ times of the average power. Due to this the efficiency of the high power amplifier reduces, because it has to generate the power at the receiver end and without any requirement, if it generating power so ultimately its efficiency will get reduced. So if the peak envelope power is subject to a design or regulatory limit, then this has the effect of reducing the mean envelope power allowed under OFDM, relative to that allowed under constant envelope modulation. It can be told that battery back-up is major important factor. So if PAPR is reduced strong battery back-up can be provided. So PAPR is basically defined as the ratio of maximum power occurring in the OFDM transmission to the average power of the OFDM transmission. [4] So mathematically it can be expressed as given below

$$
P A P R=\frac{P_{\text {peak }}}{P_{\text {average }}}=\frac{\max \left[\left|x_{n}\right|^{2}\right]}{E\left[\left|x_{n}\right|^{2}\right]}
$$

Where, $\boldsymbol{P}_{\text {peak }}=$ Peak power of the OFDM system, $\boldsymbol{P}_{\text {average }}=$ average power of the OFDM system. E $[\cdot]$ is the expectation operator [5]. So the device which is used to measure the ratio of the peak power level to the time-averaged power level is known as peak-to-average ratio meter (Par meter) 


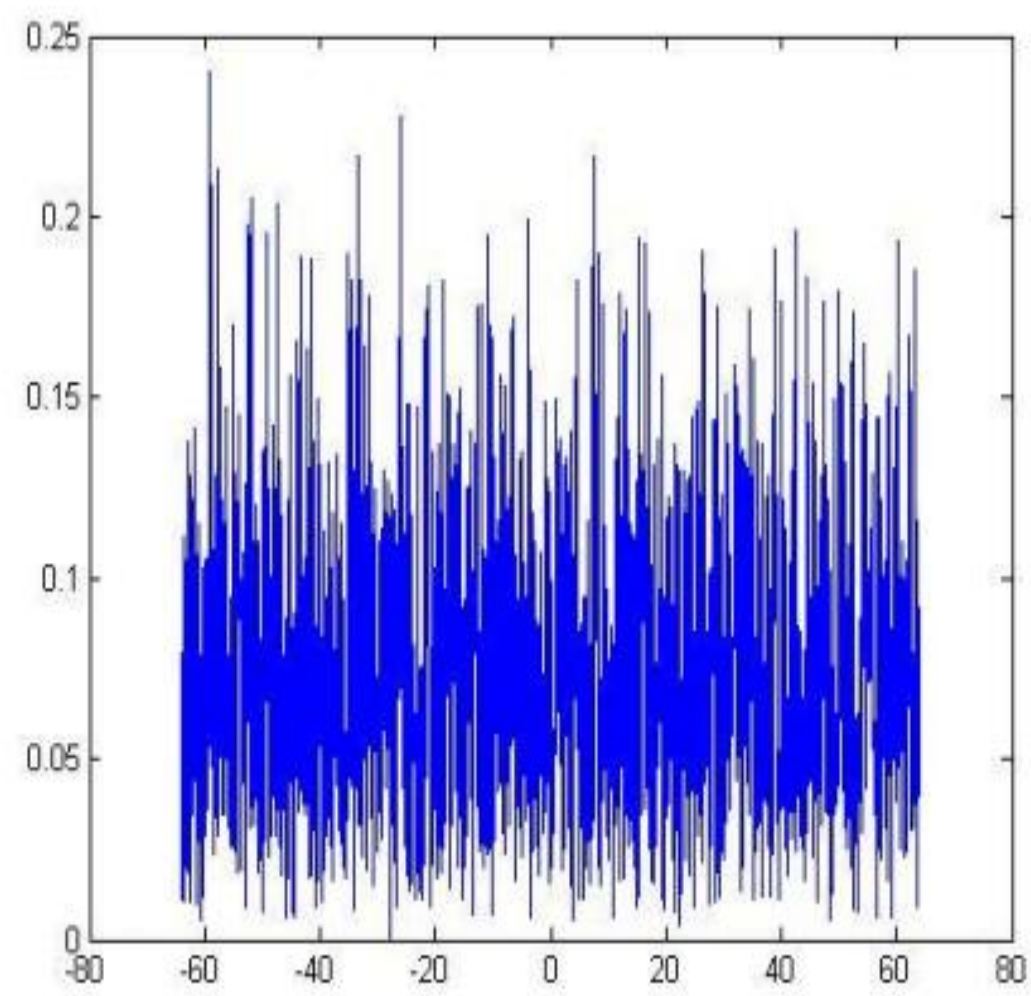

Fig.1 OFDM Signals which is containing sinusoidal high peaks [4]

\subsection{Conditions of Orthogonality:-}

It is known that two periodic signals are orthogonal when the integral of their product over one period is equal to zero. So there are two cases possible for it first is for continuous time and second is for discrete time signal.

\section{Case-1 Continuous Time:-}

$$
\int_{0}^{T} \cos \left(2 \pi n f_{0} t\right) \cos \left(2 \pi m f_{0} t\right) d t=0
$$

\section{Case-2 Discrete Time:-}

Where $m \neq n$ in both the cases, the equation for discrete time would become,

$$
\sum_{k=0}^{N-1} \cos \left(\frac{2 \pi k n}{N}\right) \cos \left(\frac{2 \pi k m}{N}\right) d t=0
$$

\subsection{Cumulative Distribution Function (CDF).}

Complementary CDF (CCDF) is used in place of CDF, which helps us to measure the probability that the PAPR of a certain data block exceeds the given threshold. Generally the CDF of the amplitude of a signal sample is given by following

$$
F(z)=1-\exp (z)
$$

CCDF of the PAPR of the data block is desired generally to compare outputs of many reduction techniques. It is given by as below

$$
\begin{aligned}
& P(P A P R>z)=1-P(P A P R \leq z) \\
& =1-F(z)^{N} \\
& \therefore P(P A P R>z)=1-\left(1-\exp \left(z^{N}\right)\right.
\end{aligned}
$$

Where $F(z)$ represent the CDF.

\section{SIMULATION RESULTS}

\section{Simulation 1}

In this simulation, $\mathrm{N}=128$ subcarriers and QPSK modulation scheme has been used. First of all OFDM has been implemented, and then signals have been clipped from a particular threshold value on matlab. At the same time BER and SNR have been maintained properly. 


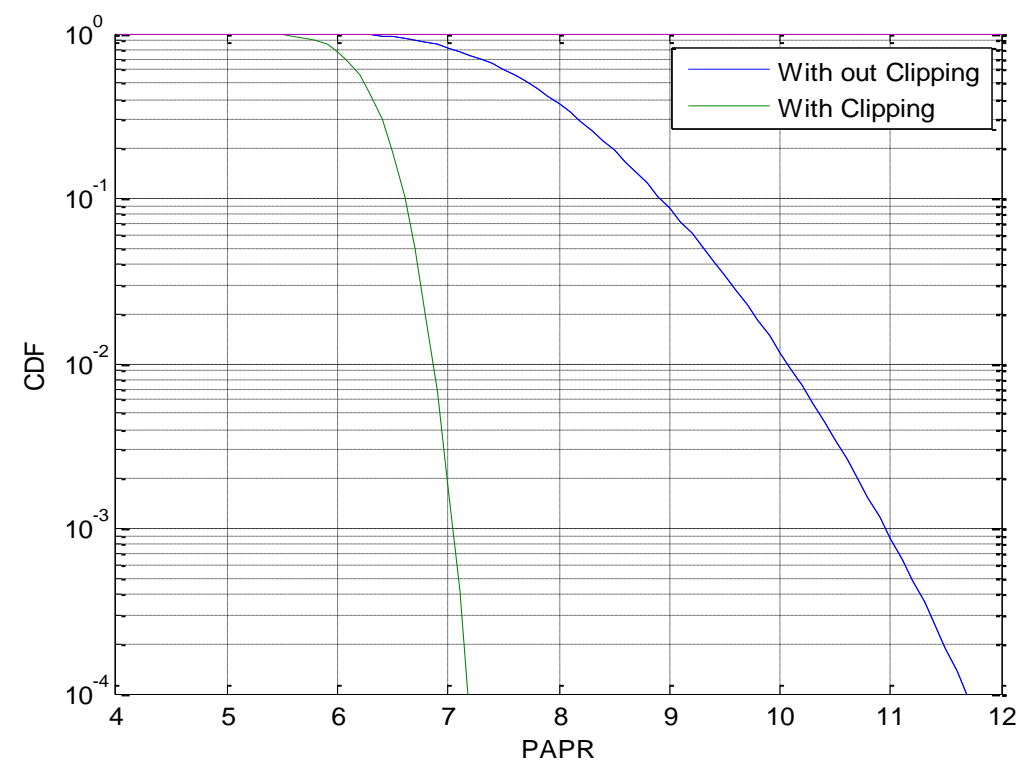

Fig.2 PAPR Vs CDF

Analysis:-In this result first of all without clipping, PAPR has been plotted, which gives the power of 11.70dB, then simple clipping technique has been used which results 7.20dB.So by using this clipping method 4.50dB of the power has been reduced. [1]

\section{PROPOSED ALGORITHM}

The main idea of the proposed method is, by implementing number of Subcarriers $\mathrm{N}=128$, Up sampling, factor $\mathrm{k}=1$, Number of all samples $n=1000000$, Mapping Order $\mathrm{M}=8$, Phase Offset $\mathrm{P}=90$ the desired PAPR has been achieved, If the up sampling factor is varying than PAPR increases and decreases, if PAPR decreases than SNR also decreases, but SNR should be high. So by implementing these above parameter, appropriate PAPR and SNR has been achieved. In up sampling and down sampling, very careful observation is required to set the sample rates, which gives lower PAPR.

\section{Simulation 2}

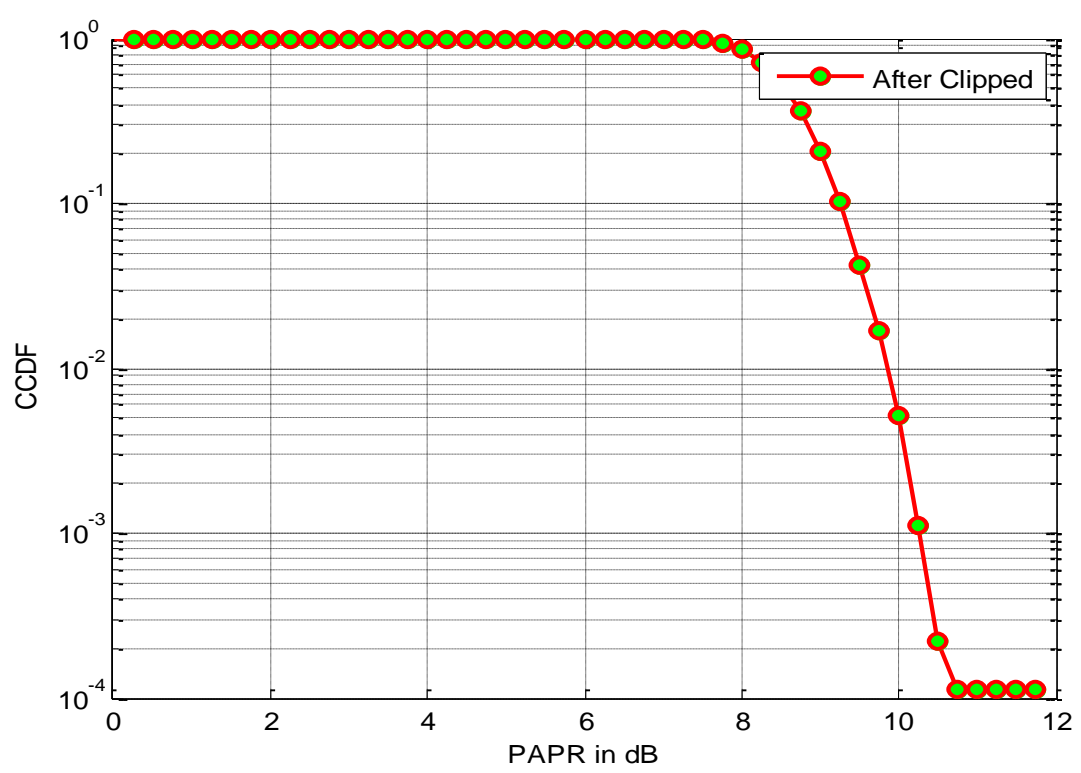

Fig.3 PAPR Vs CCDF after implementation when $\mathrm{N}=128$.

Discussion: In the fig 3, At CCDF $10^{-1}$, PAPR=9.25dB, and At CCDF 10 ${ }^{-4}$, PAPR=10.70dB. So this simulation shows better result as compare to the fig.2.In that result, At $\mathrm{CCDF} 10^{-4}$, PAPR $=11.70 \mathrm{~dB}$ but here we are getting, At CCDF $10^{-4}$, PAPR=10.70dB. So finally result is improved up to $1 \mathrm{~dB}$ due to proposed method. 


\section{Simulation 3}

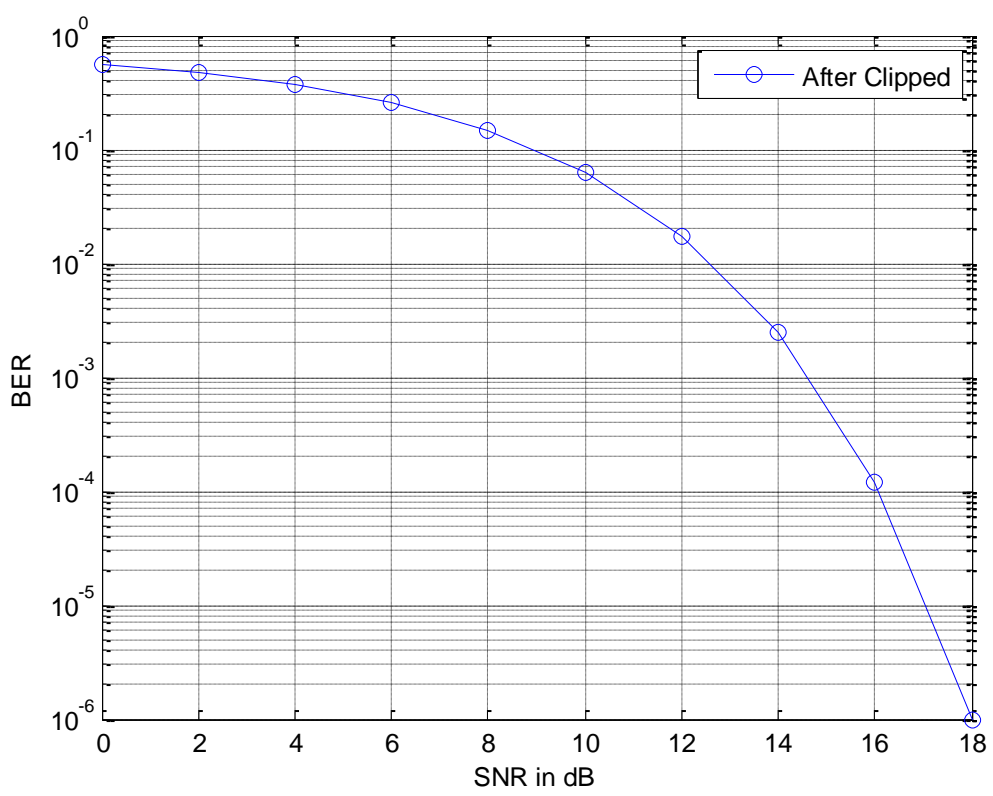

Fig.4 BER Vs SNR after implementation when $\mathrm{N}=128$

Analysis:-In the above fig. 4 , At BER $=10^{-1}, \mathrm{SNR}=9 \mathrm{~dB}$, At BER $=10^{-5}$, $\mathrm{SNR}=17 \mathrm{~dB}$ and At $\mathrm{BER}=10^{-6}$, $\mathrm{SNR}=18 \mathrm{~dB}$. It means as the (BER) bit error rate is decreasing according to that SNR (signal to noise ratio) is increasing. Theoretically BER and SNR both are inversely related and practically both are inversely related. So ultimately quality of signals is increasing. For the high quality of signal we need maximum SNR of the signal. So when SNR will be high the information capacity will be high and in case this we are getting high SNR. So final conclusion is that when BER is decreasing, then SNR is increasing and when BER is increasing then SNR is decreasing.

A. By implementing number of Subcarriers $N=512$, Up sampling, factor $\mathrm{k}=1$, Number of all samples $\mathrm{n}=1000000$, Mapping Order $\mathrm{M}=8$, Phase Offset $\mathrm{P}=90$

\section{Simulation 4}

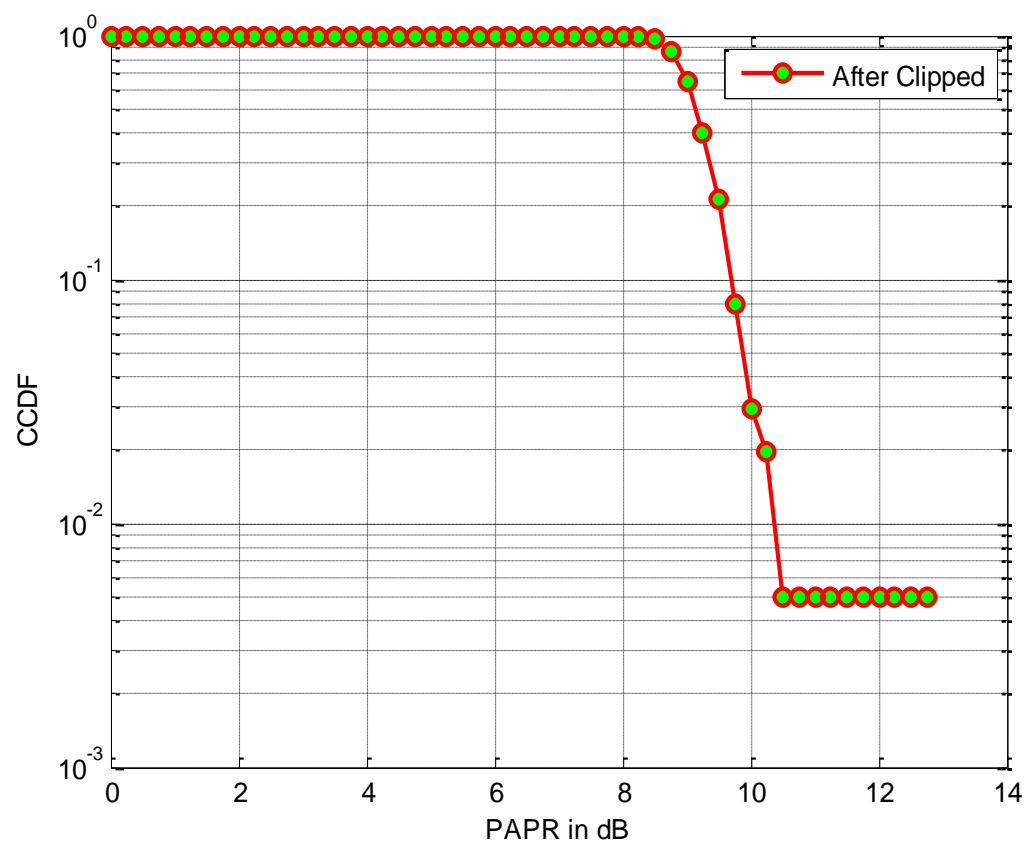

Fig.5 PAPR Vs CCDF after implementation when $\mathrm{N}=512$ 
Discussion: In the fig.5, At CCDF $10^{-1}$, PAPR $=9.75 \mathrm{~dB}$, At CCDF $10^{-3}, \mathrm{PAPR}=10.25 \mathrm{~dB}$. So this simulation also gives almost better result. As the value of $\mathrm{N}$ increases then complexity of the system increases, and PAPR also increases, this can be observe when $\mathrm{N}=256$. Ultimately, it need to trade off between value of $\mathrm{N}$ and PAPR. So finally these values should be taken such that PAPR is maintained up to desired levels.

\section{Simulation 5}

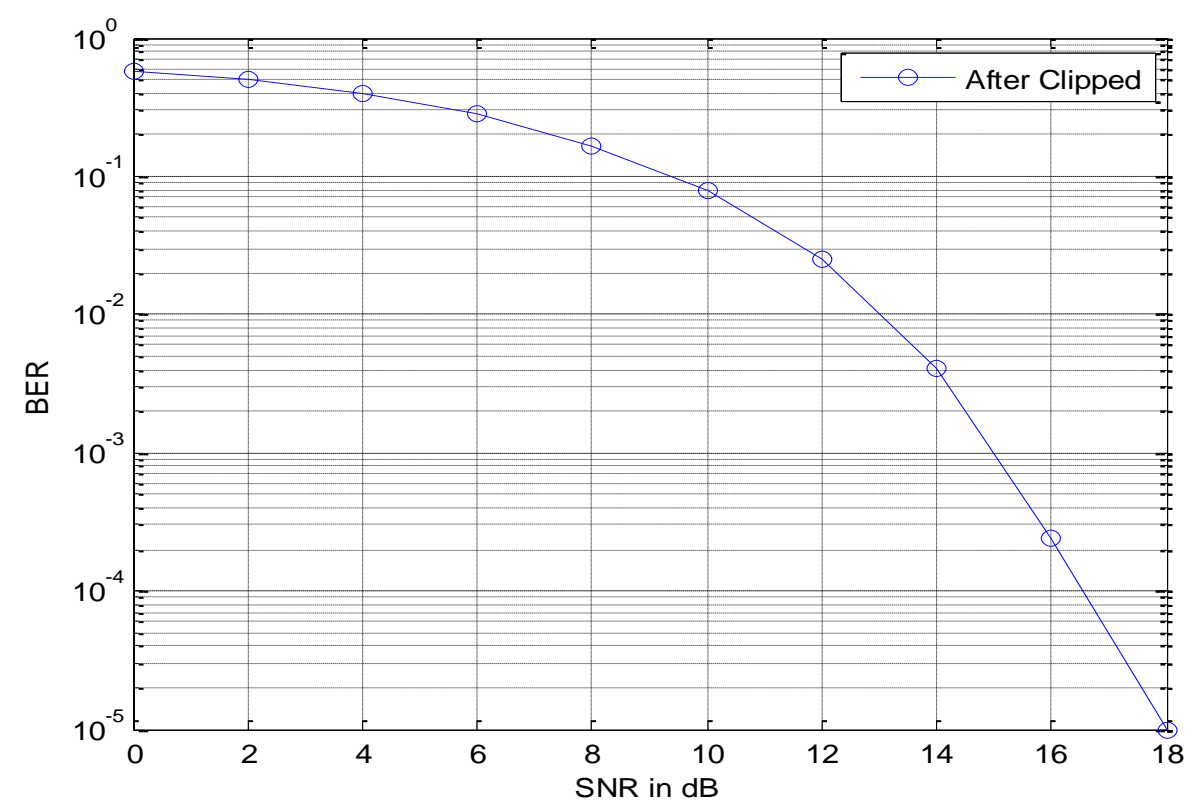

Fig.6 BER Vs SNR after implementation when $\mathrm{N}=512$

Analysis:-In the above fig.6, At BER $=10^{-1 / 2}$ (From where the graph is starting) $\mathrm{SNR}=0 \mathrm{~dB}$, and At $\mathrm{BER}=10^{-5}$, $\mathrm{SNR}=18 \mathrm{~dB}$.This is the best case where we are getting best SNR values. It means, as the BER is decreasing according to that SNR is increasing. Theoretically BER and SNR both are inversely related and practically we are getting the same relationship. So ultimately quality of signals is increasing. For the high quality of signal we need maximum SNR of the signal. So when SNR will be high the information capacity will be high and in this case we are getting high SNR. However we can say that both BER \& SNR are inversely related to each others. Ultimately, we are getting better SNR as the BER is decreasing. So finally this result shows better SNR as compare to any other cases.

\section{CONCLUSION}

OFDM systems have the generic problems of high PAPR. However the drawback of high PAPR is dynamic range of power amplifier and D/A convertor which increases its cost. Hence we apply efficient peak clipping technique to reduce PAPR. By using the proposed technique PAPR and BER has been achieved up to desired level. At the same time if the value of $\mathrm{N}$ increase at the same up sampling factor, Phase offset and mapping factor then PAPR increases but SNR decreases. But by using proposed technique desired BER i.e. minimum BER (maximum SNR) and PAPR (minimum) have been achieved. So, finally better tradeoff between BER and PAPR have been maintained.

\section{REFERENCES}

[1]. Xiaodong Zhu, Wensheng Pan, Hong Li, and Youxi Tang, Member, IEEE "Simplified Approach to Optimized Iterative Clipping and Filtering for PAPR Reduction of OFDM Signals" IEEE TRANSACTIONS ON COMMUNICATIONS, VOL. 61, NO. 5, MAY 2013

[2]. Bo Wang, Pin-Han Ho, and Chih-Hao Lin "OFDM PAPR Reduction by Shifting Null Subcarriers Among Data Subcarriers" IEEE COMMUNICATIONS LETTERS, VOL. 16, NO. 9, SEPTEMBER 2012.

[3]. John M. Senior, M.Yousif Jamro "Optical fiber communications, Principle and Practice" Pearson publication, $3^{\text {rd }}$ Edition.

[4]. Malhar Chauhan, Prof.Abhishek Chobey, "PAPR Reduction in OFDM system Using Ton Reservation Technique, ” International Journal of Computer Technology and Electronics Engineering (IJCTEE) Volume 2, Issue 4, August 2012

[5]. Kanchan Choubey, Prashant Jain, "PAPR Reduction using Companding and FEC Coding in OFDM System" International Journal of Scientific \& Engineering Research, Volume 5, Issue 1, January-2014 2150 ISSN 2229-5518 [6]. Y. Wang, L.-H, Wang, J.-H. Ge, and B. Ai, Senior Member, IEEE, "Nonlinear Companding Transform Technique for Reducing PAPR of OFDM Signals. " IEEE Transactions on Consumer Electronics, Vol. 58, No. 3, August 2012

[7] X. Huang, "companding transform for reduction in peak-to-average power ratio of OFDM signals," IEEE Trans. Wireless Commun., Vol. 03, No. 6, Nov.2004, pp. 2030 - 2039 


\section{BIOGRAPHIES}

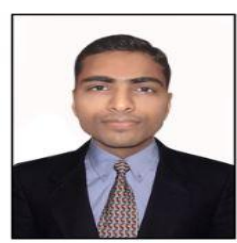

Sarvjeet Kumar is working as an Asst.

Professor in Department of ECE, S. (P.G)

.I.T.M, Rewari, Haryana. $\mathrm{He}$ has received M.Tech in Electronics and Communication Engineering. His main research includes Communication system, Antenna Engineering and Optical fiber Communication.

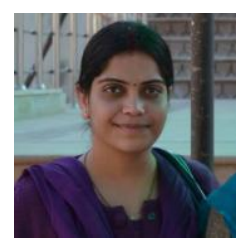

Shelly Chugh is working as an Asst.

Professor in Department of ECE, S (P.G) .I.T.M, Rewari Haryana. She has received M.Tech in Electronics \& Communication Engineering in 2012. Her main research areas are Antenna Designing and Communication Systems. 